\title{
Exploring the Effect of Long Naps on Handball Performance and Heart Rate Variability
}

(c) (1) $($ 우 $\odot$

\author{
Authors

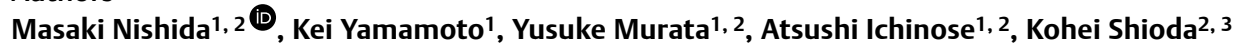

Affiliations

1 Faculty of Sport Sciences, Waseda University, Tokorozawa, Japan

2 Sleep Research Institute, Waseda University, Shinjuku-ku, Japan

3 Faculty of Human Sciences, Kanazawa Seiryo University, Kanazawa, Japan

Key words

Nap, Sleep, Sports, Handball, Heart rate variability,

Autonomic nervous system

received $\quad 28.05 .2021$

revised 29.06.2021

accepted $\quad 12.07 .2021$

Bibliography

Sports Medicine International Open 2021; 5: E73-E80

DOI 10.1055/a-1551-8054

ISSN 2367-1890

(c) 2021. The Author(s).

This is an open access article published by Thieme under the terms of the Creative Commons Attribution-NonDerivative-NonCommercial-License, permitting copying and reproduction so long as the original work is given appropriate credit. Contents may not be used for commecial purposes, or adapted, remixed, transformed or built upon. (https://creativecommons. org/licenses/by-nc-nd/4.0/).

Georg Thieme Verlag KG, Rüdigerstraße 14,

70469 Stuttgart, Germany
Correspondence

Dr. Masaki Nishida

Faculty of Sport Sciences

Waseda University, 2-579-15

359-1192 Tokorozawa

Japan

Tel.: + 81429476771 , fax: + 81429476771

nishida@waseda.jp

Supplementary Material is available under https://doi.org/10.1055/a-1551-8054.

\begin{abstract}
This study explored the effect of long naps on handball-related performance and assessed the role of the cardiac autonomic nervous system in this process. Eleven male collegiate handball players performed a repeated sequential trial consisting of a 20-m consecutive turnaround run, 10-m run with a load, and shooting the ball into a target. Participants were allocated randomly and sequentially to have a short (20 minutes) nap, long (60 minutes) nap, or no nap. The Pittsburgh Sleep Quality Index was used to assess regular sleep quality. Subjective sleepiness before and after napping was measured using the Karolinska Sleepiness Scale. Heart rate variability was recorded to assess cardiac autonomic nervous function during napping. The Pittsburgh Sleep Quality Index score was correlated with shot accuracy only after long naps $(\rho=0.636, r=0.048)$. A negative correlation was observed between the root mean square of successive differences and average load run time $(\rho=-0.929$, $p<0.001$ ). Long napping was associated with a significant benefit on performance in athletes with poor sleep quality, implying a role of the autonomic nervous system in this regard. Our findings indicate the effect of sleep quality on the endurance and resistance of handball players.
\end{abstract}

\section{Introduction}

Handball is characterized by a combination of high-and low-intensity activities, including sprints, jumps, and sudden changes of direction which demand physical strength, a rapid pace, and different types of exercising [1-3]. Fatigue has been shown to affect handball-related abilities such as maximal and rapid force development in simulated match-play settings, leading to reduced performance $[4,5]$, decreased velocity, and strength [6, 7].

A growing corpus of evidence demonstrates that sleep is an essential component of athletic performance and recovery from fa- 
tigue, demonstrating a disrupted sleep impairs sports performance by altering both cognitive and executive domains $[8,9]$. The importance of sleep in handball has been gaining attention since this sport is associated with a significantly high risk of physical injuries $[10,11]$. Napping is widely used as countermeasure to prevent sleepiness and poor performance due to sleep loss and circadian pressure. Regarding the physical performance of trained athletes, a myriad of studies supports the beneficial effects of naps on a wide array of sports performances $[12,13]$. Most of these have examined the relationship between sports performance and short naps of less than 30 minutes, which is generally accepted as a proper nap duration [14]. In recent years, additional research has been conducted to explore the effect of longer naps to compensate for chronic sleep loss, considering the apparent tendency of athletes to be sleep deprived. In this regard, it has been demonstrated that a 90-min-long nap has a beneficial effect on repeated sprint exercises and systemic biochemical responses compared to a 20-minlong nap $[15,16]$. Although these studies suggest the importance of longer naps in trained athletes, whether prolonged napping is associated with enhanced recovery and performance in athletes is still unclear.

In addition to sleep, autonomic nervous system (ANS) regulation is also involved in recovering from sustained fatigue. HRV measurements in the applied sports setting are useful to assess global changes in the ANS and determine the physiological adaptation to training in athletes $[17,18]$. Sleep and ANS activity are considered to influence each other. Changes in the ANS affect the structure and quality of sleep [19], while parasympathetic activity is likely to be modulated by sleep, even by daytime napping [20], supporting the idea that naps may be beneficial for an effective recovery, apart from compensating for chronic sleep loss [21]. Although it seems likely that nap length influences ANS activity, the exact relationship between these variables in athletes remains unclear.

Given the paucity of studies investigating the effects of nap length on ANS activity and sports performance and recovery in athletes, we conducted a study to (1) compare the sports performance after short (20 min) and long (60 min) naps, (2) assess the changes in ANS by measuring the HRV during a daytime nap, and (3) explore the relationship between HRV and napping duration and their impact on sports performance. We hypothesized that a long nap is beneficial for handball-related performance compared with no nap or a short nap, involving the increased activity of the parasympathetic nervous system during sleep.

\section{Materials and Methods}

\section{Participants}

Participants were recruited from the male handball team of Waseda University Club, which belongs to the first division of the Japan University Handball League. Exclusion criteria were: (1) less than one year of handball career, (2) prior diagnosis of a pathological sleep disorder, (3) use of any medication. Eventually, 11 of 40 team members, (age: $20.7 \pm 1.2$ years, height: $174.9 \pm 4.9 \mathrm{~cm}$, body mass index: $24.3 \pm 1.1$ ) consented to participate in this study. Participants were requested to keep a normal sleep-wake rhythm during the three days before the experiment. Additionally, they were asked to refrain from consuming caffeine on the day of the experiment.

The Academic Research Ethical Review Committee of Waseda University approved all activities (IRB \#2020-270) and participants provided informed consent before any procedure took place, which was conducted in accordance with the 1964 Declaration of Helsinki. Additionally, we confirmed that the current study meets the ethical standards of the journal [22].

\section{Experimental design}

The experiment protocol applied a crossover repeated-measures design consisting of three test sessions. After a familiarization session, participants were randomly assigned three nap opportunity conditions: no nap (0 min), a short nap (20 min), and a long nap (60 $\mathrm{min})$ in random order with at least a 1-week interval between each. Participants were asked not to engage in strenuous exercise before the experiment. At 12:45 pm, participants entered a quiet, air-conditioned, dark room near the gymnasium where the temperature was set at approximately $25.0^{\circ} \mathrm{C}$. Participants sat deeply in a soft beanbag chair (Yogibo MAX, Webshark Inc., Osaka, Japan) and were requested to take a nap after the lights were turned off at 13:00. Then, they were woken up by an examiner (KY) at 13:20 in the case of short naps and at 14:00 in the case of long naps. Individuals undergoing the no-nap condition stayed at the gymnasium and were asked to strictly avoid active movements and oral intake. Considering the decreased vigilance after wakeup (conceptualized as 'sleep inertia'), handball performance trials were conducted at 16:30, the usual training time.

\section{Sleep assessments}

Subjective sleep evaluation was performed by standardized questionnaire. Objective sleep evaluations for nocturnal sleep on the day before the experiment and for napping on the day of the experiment were conducted using two different devices. The regular sleep quality was assessed using the Pittsburgh Sleep Quality Index (PSQI), which is a practical self-reported questionnaire measure of sleep and incorporates qualitative and quantitative aspects of sleep [23] The Morningness-Eveningness Questionnaire (MEQ) was evaluated to measure chronotype that is defined as the time of day preferred by individuals to perform daily activities, indicating higher scores of MEQ as morningness [24]. The level of sleepiness was rated using the Karolinska Sleepiness Scale (KSS), which is used as a momentary assessment of a state of sleepiness, before and after naps. When no naps were taken, the KSS was assessed at a similar time as if these were taken. A visual analog scale (VAS) was used for the subjective evaluation of sleep quality (0: poor; 10: excellent).

To evaluate sleep the night before the experiment, a wearable device (Fitbit Charge3, Fitbit Inc., San Francisco, CA, USA) was attached to the participants. Fitbit models differentiated sleep from wakefulness using the similar technology to actigraphy known as an accelerometer. Although scoring sleep stage of Fitbit software has not been guaranteed to be as accurate as polysomnography, the performance in differentiating wake from sleep epochs is better than that reported for actigraphy [25]. Sleep evaluation during napping was performed using a different device described later. 


\section{Experimental handball performance}

The experimental performance was devised in line with the practical play of handball referring to previous review [26]; it comprised three different tasks: 1) a 20-m turnaround run, 2) a 10-m run carrying a load, and 3 ) shooting the ball three times into a target ( Fig. 1.). Participants were instructed to repeat the series of tasks four times uninterruptedly. All tasks were performed in the university gymnasium and were supervised carefully by an examiner (KY). Participants did not perform warm-up for preparatory exercises.

Three cones were placed 10 meters apart in a straight line to cover a total distance of 20 meters. Participants were instructed to perform three and a half turnaround runs at full speed. During the last 10 meters, the participant carried a person of similar weight, varying in the $\pm 3 \mathrm{~kg}$ range of their own weight on his back. Finally, at the level of the second cone and while still moving, participants made three shots with the handball into a $45 \mathrm{~cm}$-sized square target.

We recorded the times of each turnaround run and the last 10meter load run. For evaluating shooting accuracy, the experimenter visually checked whether it was successful or not. We used the average of these data for the subsequent analysis involving nap conditions.

\section{Sleep (nap) and ANS function evaluation}

For assessing sleep propensities and heart rate during napping, Actiheart 5 monitors (Cambridge Neurotechnology, Cambridge, UK) were used to obtain continuous electrocardiogram and motility recordings, as well as sleep parameters. Data recorded for heart rate and sleep were retrieved for analysis. The Actiheart 5 is a lightweight (10.5 g) wearable device for which high intra-and inter-instrument reliability and accuracy have been reported [27].

For HRV analysis, interbeat interval data was processed using the Actiheart software (version 5.0.5) using 5-min epochs for both the time and frequency domains (using fast Fourier transform). Artifacts interpreted as invalid data were excluded from analysis ac- cording to the discrimination set by the Actiheart software. Heart rate variability was indexed in analyses using the root mean square of successive differences (RMSSD) and according to high frequency (HF; $0.15-0.4 \mathrm{~Hz})$ and low frequency $(\mathrm{LF} ; 0.04-0.15 \mathrm{~Hz})$ as well as the LF/HF ratio. The 5 -min epochs obtained during naps were averaged to generate an aggregate value. In addition, sleep analysis was conducted based upon activity obtained from motion data during naps retrieved by the monitor and provided by the software to yield sleep variables.

\section{Statistical analysis}

Demographic results are expressed as mean \pm standard deviation $(\mathrm{M} \pm \mathrm{SD})$ and variables of performance and $\mathrm{HRV}$ as mean \pm the standard error of the mean $(\mathrm{M} \pm \mathrm{SEM})$. To compare variables with nap propensities between shorter and longer nap conditions, we used a Wilcoxon signed-rank test. Napping conditions (including the no-nap condition) were compared by using Friedman's nonparametric analysis of variance. To assess differences of performance across sequential trials between napping conditions, linear mixed effect (LME) models were applied, as these enable effective use of all information, even that from participants with missing data, increasing the overall statistical power. LME allows the influence of factors for which data are extracted randomly from a population, providing more reliable results [28]. To examine handballrelated performance according to different napping opportunities, a model was built using "participant" as a random effect and "condition" (no nap, short nap, and long nap) as a fixed effect. Bonferroni correction for multiple comparisons was used during post-hoc analysis if the association was deemed significant.

Spearman's rank-order correlations were calculated to examine the association between task performance, PSQI, and the indices obtained by HRV analysis. All calculations were performed using (SPSS Advanced Statistics version 26, IBM Corp., Armonk, NY, USA).

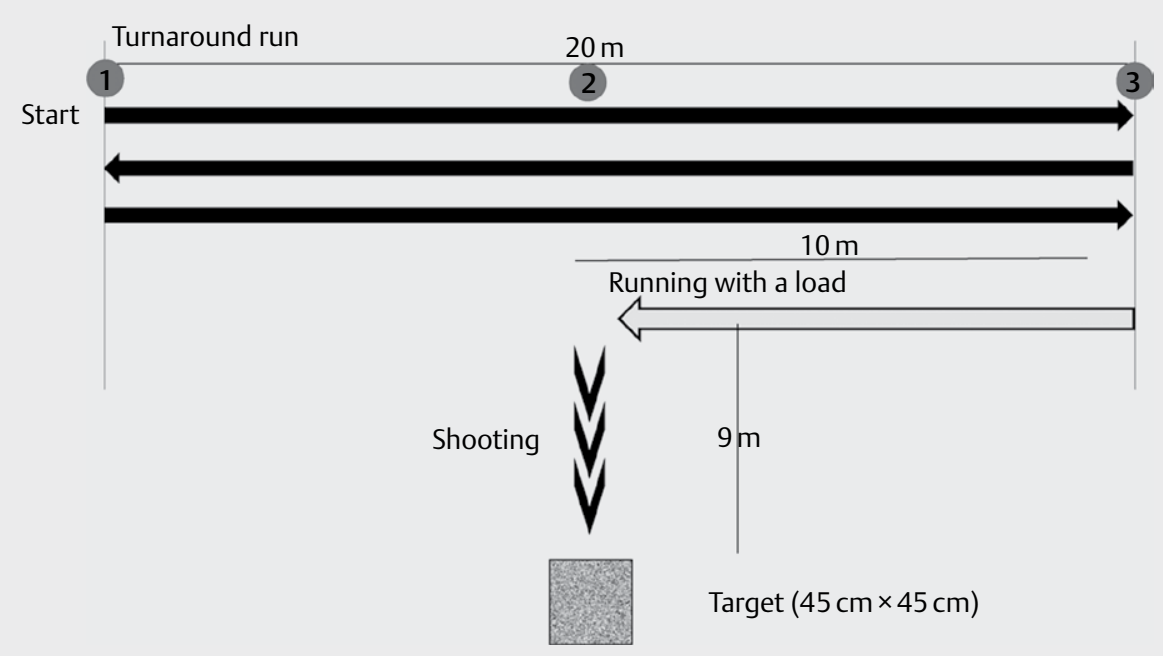

- Fig. 1 Diagram of the experimental handball performance. First, three 20-m turnaround sprints were performed. Then, a 10-meter run was performed while carrying a person of similar weight on the back. Finally, three handball shots were attempted into a $45 \mathrm{~cm}$ square goal target. 


\section{Results}

\section{Sleep parameters}

Sleep parameters of naps and of the preceding night are shown in - Table 1. Data from a nap condition of one participant was excluded due to difficulty napping. Sleep variables of the preceding night of the experiment exhibited no statistical difference among the three napping conditions. Apart from an expected difference in nap duration, none of the sleep parameters showed a significant difference between the short and long napping conditions. The KSS scores were similar between the three napping conditions.

\section{Physical performance associated with nap conditions}

The aspects comprising handball-related performance were explored according to different napping conditions ( $>$ Table 2 ). Nap conditions and trials showed no significant interaction effects for the $20-\mathrm{m}$ turnaround run $(F(2,23)=1.192, p=0.322), 10$ - $\mathrm{m}$ load run $(F(2,23)=$ $0.094, p=0.911)$, and shooting accuracy $(F(2,23)=2.348, p=0.118)$. Similarly, no significant condition effect was observed for the $20-\mathrm{m}$ turnaround run $(F(2,23)=1.418, p=0.263), 10$ - $\mathrm{m}$ load run $(F(2,23)=$ $0.211, p=0.811)$, and shooting accuracy $(F(2,23)=2.148, p=0.140)$ ( Supplementary Table 1).

\section{Association between sleep parameters and physical performance}

- Table $\mathbf{3}$ and $>$ Fig. $\mathbf{2}$ demonstrate the correlation between sleep parameters and physical performance. Regarding the association between PSQI score and handball-related performance according to each napping condition, the average shooting accuracy was correlated with PSQI score in the longer nap condition $(\rho=0.658$, $p=0.039$ ), whereas this correlation did not occur in the short nap condition $(\rho=-0.493, p=0.117)$. No other significant correlations were found between the PSQI score and the remaining performance factors in either the short or long nap conditions. MEQ scores showed no significant correlation with performance variables in each napping condition.

\section{HRV analysis and physical performance}

There was no statistical difference regarding HRV parameters between the short and long nap conditions ( $\triangleright$ Table 4). Regarding correlation analysis between HRV variables and physical performance, RMSSD showed a negative correlation with the average $10-\mathrm{m}$ load run time in the long nap condition $(\rho=-0.929, p<0.001)$ ( $\triangleright$ Fig. 3). In contrast, no significant correlation between HRV indices and physical performances was found in the short nap condition.

- Table 2 Handball-related performance outcomes.

\begin{tabular}{|l|l|l|l|}
\hline Variable measured & No nap & Short nap & Long nap \\
\hline 20 -m turnaround run $(\mathrm{s})$ & $12.36(0.07)$ & $12.08(0.27)$ & $12.23(0.31)$ \\
\hline $1^{\text {st }}$ trial & $12.54(0.24)$ & $12.51(0.29)$ & $12.58(0.29)$ \\
\hline $2^{\text {nd }}$ trial & $12.58(0.14)$ & $12.50(0.21)$ & $12.64(0.17)$ \\
\hline $3^{\text {rd }}$ trial & $12.35(0.20)$ & $12.30(0.27)$ & $12.64(0.24)$ \\
\hline $4^{\text {th }}$ trial & $12.46(0.18)$ & $12.35(0.24)$ & $12.52(0.24)$ \\
\hline Average & $5.36(0.22)$ & $5.67(0.22)$ & $5.35(0.18)$ \\
\hline 10 -m load run $(\mathrm{s})$ & $5.55(0.18)$ & $5.48(0.24)$ & $5.45(0.18)$ \\
\hline $1^{\text {st }}$ trial & $5.48(0.17)$ & $5.51(0.20)$ & $5.35(0.18)$ \\
\hline $2^{\text {nd }}$ trial & $5.48(0.15)$ & $5.41(0.22)$ & $5.20(0.10)$ \\
\hline $3^{\text {rd }}$ trial & $5.46(0.16)$ & $5.52(0.19)$ & $5.34(0.13)$ \\
\hline $4^{\text {th }}$ trial & $56.7(11.2)$ & $74.1(7.0)$ & $50.0(7.5)$ \\
\hline Average & $60.0(13.0)$ & $66.7(9.1)$ & $66.7(8.6)$ \\
\hline Accuracy of shooting $(\%)$ & $66.7(11.1)$ & $59.3(11.4)$ & $73.4(8.3)$ \\
\hline $1^{\text {st }}$ trial & $76.7(7.1)$ & $66.7(9.1)$ & $76.7(8.7)$ \\
\hline $2^{\text {nd }}$ trial & $65.0(5.2)$ & $66.7(5.4)$ & $66.7(5.0)$ \\
\hline $3^{\text {rd }}$ trial & \multicolumn{5}{|l}{} \\
\hline $4^{\text {th }}$ trial & Average & \multicolumn{5}{|l}{} \\
\hline Values are shown as mean (standard error of the mean). \\
\hline
\end{tabular}

- Table 1 Sleep-related variables.

\begin{tabular}{|c|c|c|c|c|c|}
\hline Variable measured & No nap & Short nap & Long nap & Z-value & p-value \\
\hline \multicolumn{6}{|l|}{ Experimental nap } \\
\hline Total time on sofa (min) & & $31.0(1.9)$ & $64.0(4.8)$ & -2.530 & 0.011 \\
\hline Total sleep time (min) & & $28.0(5.7)$ & $57.9(7.5)$ & -2.521 & 0.012 \\
\hline Sleep onset latency (min) & & $3.4(4.4)$ & $6.1(4.8)$ & -0.986 & 0.324 \\
\hline Sleep efficiency (\%) & & $93.9(4.3)$ & $96.2(1.7)$ & -1.260 & 0.208 \\
\hline KSS score before nap & & $5.22(1.39)$ & $4.56(2.30)$ & -0.923 & 0.356 \\
\hline KSS score after nap & $5.33(1.80)$ & $4.89(2.37)$ & $4.80(2.30)$ & & 0.292 \\
\hline Subjective sleep quality (VAS) & & $6.67(1.22)$ & $6.89(1.36)$ & -0.357 & 0.726 \\
\hline \multicolumn{6}{|l|}{ Night prior to the experiment } \\
\hline Total sleep time (h) & $7.60(0.67)$ & $7.00(0.79)$ & $7.33(1.15)$ & & 0.309 \\
\hline Latency to sleep onset (m) & $5.89(2.09)$ & $6.44(2.35)$ & $7.00(0.79)$ & & 0.549 \\
\hline Sleep efficiency (\%) & $90.4(4.2)$ & $91.1(4.9)$ & $92.2(2.6)$ & & 0.439 \\
\hline
\end{tabular}




\section{Discussion}

The main findings of the current study were as follows: (1) no significant effect was found between napping conditions and trials in handball-related tasks, and (2) longer naps were likely more beneficial for 10-m turnaround runs than shorter naps and improved

- Table 3 Correlations between the PSQI and MEQ scores and performance outcomes in short and long nap conditions

\begin{tabular}{|l|l|l|c|c|}
\hline & \multicolumn{2}{|c|}{ Short nap } & \multicolumn{2}{c|}{ Long nap } \\
\hline & PSQI & MEQ & PSQI & MEQ \\
\hline KSS (pre-nap) & -0.064 & -0.496 & 0.079 & -0.009 \\
\hline KSS (post-nap) & -0.247 & -0.513 & 0.511 & -0.455 \\
\hline VAS & -0.082 & 0.492 & 0.397 & 0.506 \\
\hline $\begin{array}{l}20-m \text { turnaround run } \\
\text { (averaged) }\end{array}$ & -0.345 & 0.050 & -0.064 & -0.059 \\
\hline $\begin{array}{l}10-m \text { load run } \\
\text { (averaged) }\end{array}$ & 0.400 & -0.180 & 0.295 & -0.371 \\
\hline Accuracy (averaged) & -0.493 & -0.485 & $0.658^{*}$ & 0.161 \\
\hline
\end{tabular}

PSQI, Pittsburgh Sleep Quality Index; MEQ, morningness-eveningness questionnaire; KSS, Karolinska Sleepiness Scale; VAS, Visual Analogue Scale. The coefficient and significance values were obtained using the Spearman rank-order test. ${ }^{*} \mathrm{p}=0.039$ ball shooting accuracy in individuals with high PSQI scores. Furthermore, (3) the novelty of this study was that RMSSD, which reflects parasympathetic function, correlated negatively with the $10-\mathrm{m}$ load run time only in the long nap condition.

Several studies have demonstrated the beneficial effect of long naps on physical performance, even in individuals without sleep deprivation. For instance, Boukhris et al. demonstrated that a 90min nap opportunity resulted in enhanced attention, improved maximal voluntary isometric contraction, and longer distance of shuttle sprints compared with 40-min and no-nap opportunities [29]. In contrast, our study did not observe these correlations. Conversely, Suppiah et al. reported no beneficial effects of daytime short nap on sprint and shooting performance among Asian youth elite performance athletes [30]. The authors attributed inconsistency to chronically sleep-deprived young collegiate athletes who cannot compensate for a habitual lack of sleep with a one-day napping intervention. Another study demonstrated that Japanese student athletes tend to be sleep-deprived, and lifestyle habits, mental health, and competition activities are closely related to sleep deterioration [31]. Accordingly, sleep is greatly affected by the social environment, such as dwelling or commute, as reported in a previous study with similar populations [32]. Moreover, studies have estimated that the detrimental effects of chronic sleep dep-
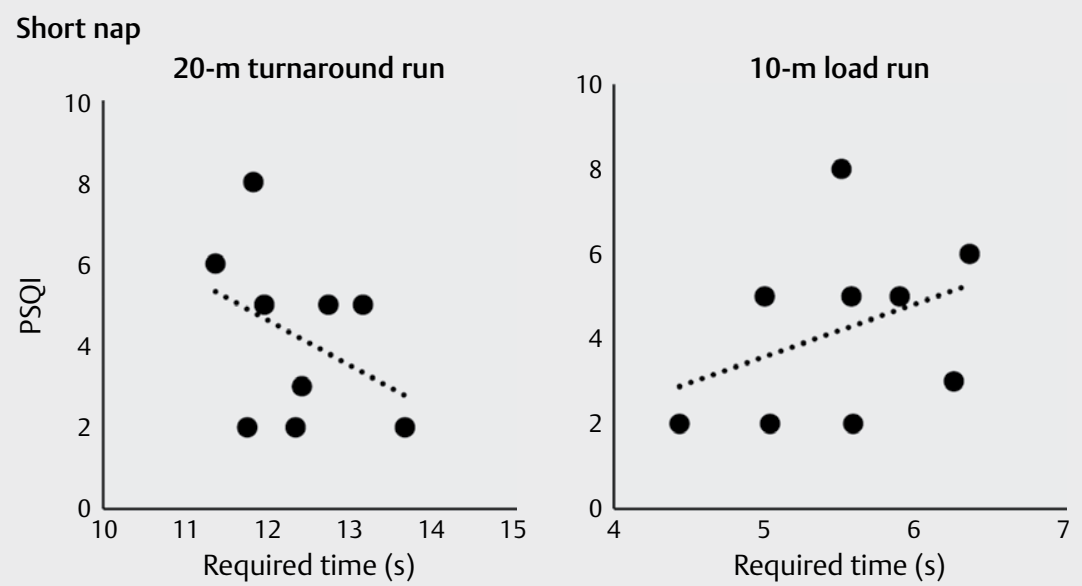

Ball shooting
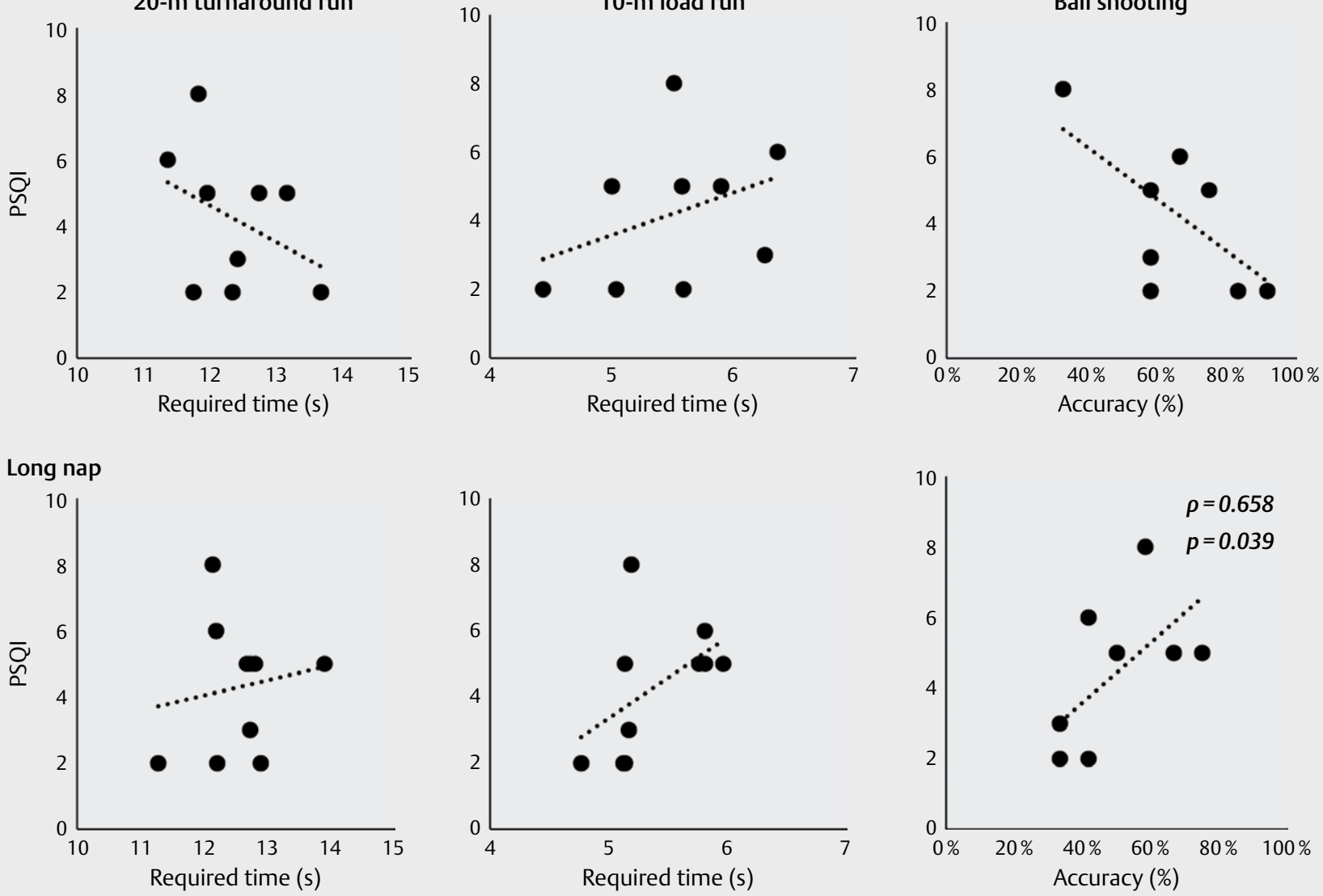

- Fig. 2 Correlation between the PSQI score and athletic performance in the short and long nap conditions. The $y$-axis displays the PSQI score and the $x$-axis displays the performance outcomes. Higher PSQI scores indicate poorer sleep quality. PSQI, Pittsburgh Sleep Quality Index. 
- Table 4 Heart rate variability indices during naps.

\begin{tabular}{|l|l|l|l|l|}
\hline & Short nap & Long nap & Z-value & p-value \\
\hline Heart rate (bpm) & $63.3(3.4)$ & $65.9(1.4)$ & -0.980 & 0.327 \\
\hline RMSSD (msec) & $70.6(47.9)$ & $73.4(7.0)$ & -1.120 & 0.263 \\
\hline HF & $1009.1(314.9)$ & $1073.4(233.1)$ & -0.000 & 1.000 \\
\hline LF & $1914.1(340.9)$ & $1978.0(333.6)$ & -0.560 & 0.575 \\
\hline LF/HF index & $2.54(0.62)$ & $2.41(0.77)$ & -0.560 & 0.575 \\
\hline
\end{tabular}

RMSSD, root mean square of successive differences; HF, high frequency; LF, low frequency. Values are shown as mean (standard error of the mean).

Significance was assessed using the Wilcoxon signed rank test.

\section{Short nap}
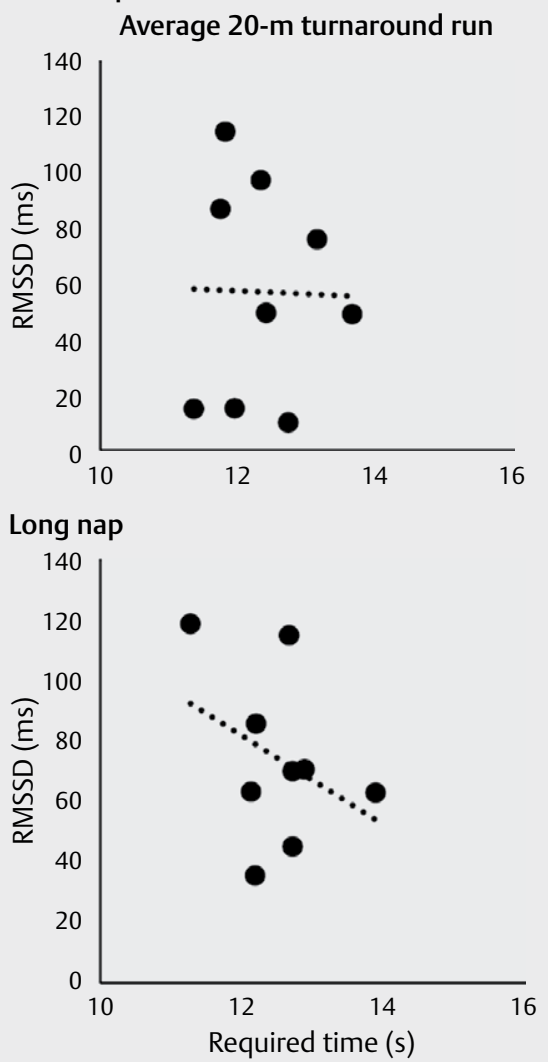
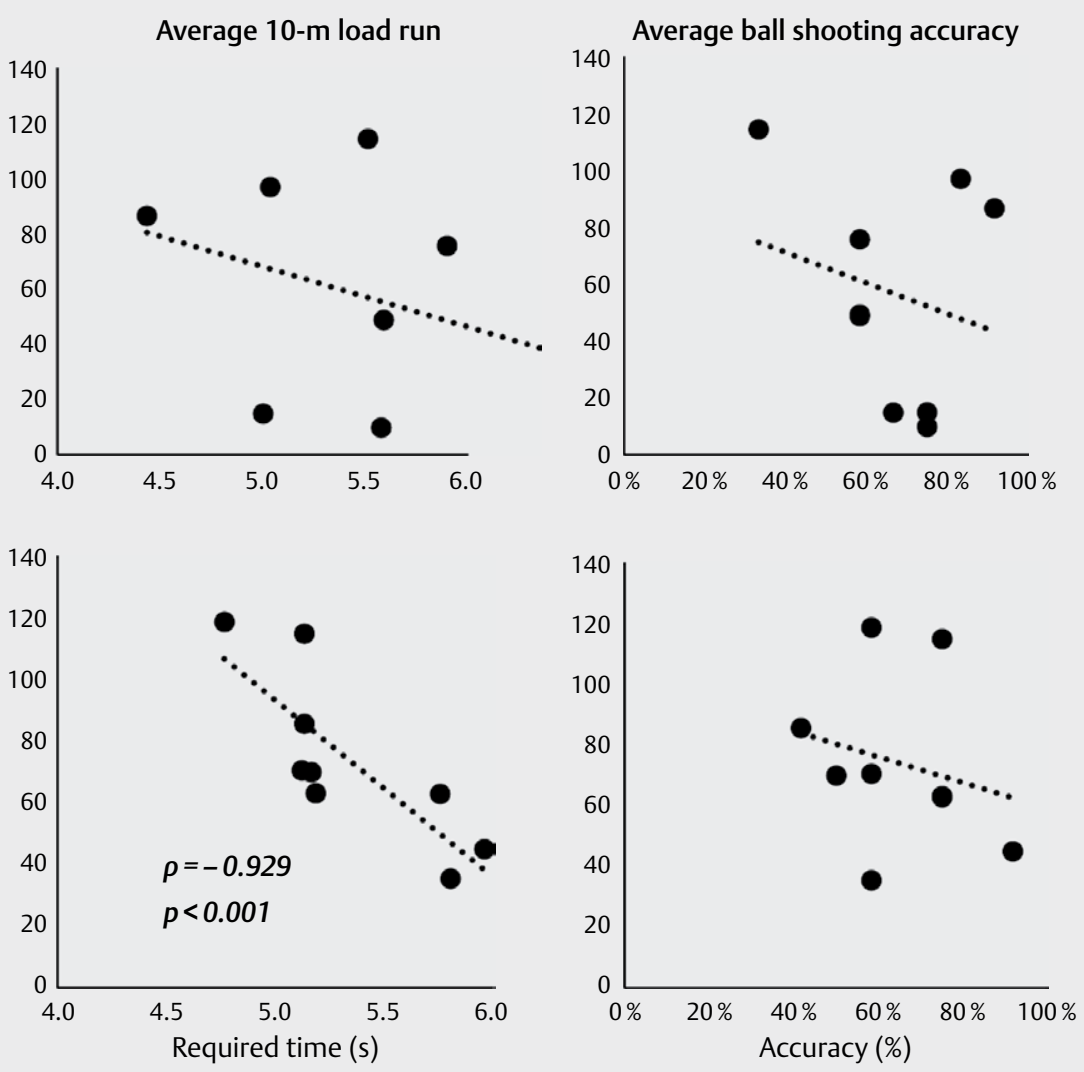

- Fig. 3 Correlation between RMSSD and performance outcomes in short and long napping conditions. The $y$-axis displays the RMSSD value, and the $x$-axis displays the average performance outcome values. RMSSD, root mean square of successive differences.

rivation [33, 34]. Thus, a single nap, regardless of duration, may not result in a significant change in performance.

Our results showed that long naps were associated with an improved ball shooting performance in participants with high PSQI scores. This implies that athletes with an inadequate sleep quality can benefit from long naps. It is generally agreed that sleep loss deteriorates a wide range of sports performances [35]. Increasing evidence suggests that an extended sleep, with the purpose of reducing or eliminating chronic sleep deprivation, improves performance in various sports including basketball, tennis, and enduring cycling $[34,36,37]$. Additionally, polysomnographic investigations reveal that daytime napping is also effective for supplementing nighttime sleep and maintaining sleep structure, representing an effective strategy to counteract sleep loss in athletes [21]. Therefore, long naps may be particularly beneficial in sleep-deprived athletes.

HRV analysis revealed that the perceived recovery from fatigue and 10-m load run (high-load task) performance were associated with ANS alterations, as assessed by the RMSSD. The RMSSD is an accepted marker of parasympathetic activity that represents a practical measure of HRV, as higher RMSSD indicates enhanced cardiac-parasympathetic activity [38]. This result is consistent with those of previous research demonstrating that significant increments in the training load result in decrements in parasympathetic-mediated HRV in a variety of athletes [39-42]. Regarding ANS 
activity during sleep, Chen et al. [43] have shown that HRV during NREM (non-rapid eye movement) sleep is driven mainly by parasympathetic activity. As longer naps are likely to include REM (rapid eye movement) sleep as well, there may be some disadvantages to extending naps concerning ANS regulation.

Limitations of the present study include the small number of participants and the fact that these were exclusively men. In this regard, previous studies evaluating actigraphy recordings have demonstrated that female collegiate athletes present better sleep quality than their male counterparts $[14,44]$. Therefore, our results cannot be extrapolated to women. Another limitation lay in the difficulty of setting a handball-related task. For instance, ball shooting was employed several times, but did not elucidate the demand for considerable concentration during competition. There was also the issue of habituation. Finally, even with averaged trials, the performance metrics are likely to depend substantially on day-to-day variability, a fact that significantly limits the generalizability of these outcomes. Further research is warranted to investigate the role of napping on psychological and physical performance, as well as on ameliorating the mental health of athletes since sleep disturbance and autonomic nervous system function are closely associated with mental disorders.

\section{Acknowledgements}

The authors would like to thank to all the members and staff of the Waseda University handball club.

\section{Funding}

This work was supported by the JSPS KAKENHI under Grant number JP18K10834 and JP21K11458.

\section{Conflict of Interest}

The author (MN) has received research grant from Webshark Inc.

\section{References}

[1] Wagner $\mathrm{H}$, Fuchs $\mathrm{P}$, Fusco A et al. Physical performance in elite male and female team handball players. Int I Sports Physiol Perform 2019; 14: 60-67. doi: 10.1123/ijspp.2018-0014

[2] Achenbach L, Krutsch V, Weber J et al. Neuromuscular exercises prevent severe knee injury in adolescent team handball players. Knee Surg Sports Traumatol Arthrosc 2018; 26: 1901-1908. doi: 10.1007| s00167-017-4758-5

[3] Fieseler G, Hermassi S, Hoffmeyer B et al. Differences in anthropometric characteristics in relation to throwing velocity and competitive level in professional male team handball: a tool for talent profiling. J Sports Med Phys Fitness 2017; 57: 985-992. doi: 10.23736/S00224707.17.06938-9

[4] Michalsik LB, Madsen K, Aagaard P. Physiological capacity and physical testing in male elite team handball. J Sports Med Phys Fitness 2015; 55: $415-429$
[5] Thorlund JB, Michalsik LB, Madsen K et al. Acute fatigue-induced changes in muscle mechanical properties and neuromuscular activity in elite handball players following a handball match. Scand J Med Sci Sports 2008; 18: 462-472 . doi: 10.1111/j.1600-0838.2007.00710.x

[6] Andrade MS, de Carvalho Koffes F, Benedito-Silva AA et al. Effect of fatigue caused by a simulated handball game on ball throwing velocity, shoulder muscle strength and balance ratio: a prospective study. BMC Sports Sci Med Rehabil 2016; 8: 13-19. eCollection 2016. doi: 10.1186/s13102-016-0038-9

[7] Nuno A, Chirosa IJ, van den Tillaar R et al. Effects of fatigue on throwing performance in experienced team handball players. J Hum Kinet 2016; 54: 103-113. doi: 10.1515/hukin-2016-0039

[8] Malhotra RK. Sleep, recovery, and performance in sports. Neurol Clin 2017; 35: 547-557. doi: 10.1016/j.ncl.2017.03.002

[9] Watson AM. Sleep and athletic performance. Curr Sports Med Rep 2017; 16: 413-418. doi: 10.1249/JSR.0000000000000418

[10] Raya-González J, Clemente FM, Beato M et al. Injury profile of male and female senior and youth handball players: A systematic review. Int J Environ Res Public Health 2020; 17: 3925. doi:10.3390/ijerph17113925

[11] Salzer S, Heansel R, Rey J et al. Injury occurrence in male handball players and its impact on physical constitution. Int J Sports Med 2020; 41: 328-338. doi: 10.1055/a-1028-7630

[12] Gupta L, Morgan K, North C et al. Napping in high-performance athletes: Sleepiness or sleepability?. Eur J Sports Sci 2021; 21: 321-330

[13] Stephenson KL, Trbovich AM, Vandermark LW et al. Exploring the effect of napping on sleep quality and duration in collegiate athletes. J Am Coll Health 2020; 19: 1-6. doi: 10.1080/07448481.2020.1803881

[14] Petit E, Mougin F, Bourdin H et al. A 20-min nap in athletes changes subsequent sleep architecture but does not alter physical performances after normal sleep or 5-h phase-advance conditions. Eur J Appl Physiol 2014; 114: 305-315 doi: 10.1007/s00421-013-2776-7

[15] Romdhani M, Souissi N, Chaabouni Y et al. Improved physical performance and decreased muscular and oxidative damage with postlunch napping after partial sleep deprivation in athletes. Int J Sports Physiol Perform 2020; 15: 874-883. doi: 10.1123/ ijspp.2019-0308

[16] Hammouda O, Romdhani M, Chaabouni Y et al. Diurnal napping after partial sleep deprivation affected hematological and biochemical responses during repeated sprint. Biol Rhythm Res 2018; 49: 927-939. doi.10.1080/09291016.2018.1429553

[17] Schmitt L, Regnard J, Parmentier AL et al. Typology of "fatigue" by heart rate variability analysis in elite Nordic-skiers. Int J Sports Med 2015; 36: 999-1007. doi: 10.1055/s-0035-1548885

[18] Flatt AA, Esco MR, Nakamura FY. Association between subjective indicators of recovery status and heart rate variability among Divison-1 sprint-swimmers. Sports (Basel) 2018; 6: doi: 10.3390/ sports6030093

[19] Trinder J, Waloszek J, Woods MJ et al. Sleep and cardiovascular regulation. Pflugers Arch 2012; 463: 161-168. doi: 10.1007| s00424-011-1041-3

[20] Cellini N, Whitehurst LN, McDevitt EA et al. Heart rate variability during daytime naps in healthy adults: Autonomic profile and short-term reliability. Psychophysiology 2016; 53: 473-481. doi: 10.1111/psyp. 12595

[21] Romyn G, Lastella M, Miller D] et al. Daytime naps can be used to supplement night-time sleep in athletes. Chronobiol Int 2018; 35 : 865-868. doi: 10.1080/07420528.2018.1466795

[22] Harriss D], MacSween A, Atkinson G. Ethical standards in sport and exercise science research: 2020 update. Int J Sports Med 2019; 40: 813-817. doi: 10.1055/a-1015-3123 
[23] Buysse D], Reynolds CF, Monk TH et al. The Pittsburgh Sleep Quality Index: a new instrument for psychiatric practice and research. Psychiatry Res 1989; 28: 193-213. doi: 10.1016/01651781(89)90047-4

[24] Horne JA, Ostberg O. A self-assessment questionnaire to determine morningness-eveningness in human circadian rhythms. Int J Chronobiol 1976; 4: 97-110

[25] Haghayegh S, Khoshnevis S, Smolensky MH et al. Accuracy of wristband Fitbit models in assessing sleep: systematic review and meta-analysis. J Med Internet Res 2019; 21: e16273. doi: $10.2196 / 16273$

[26] Wagner $\mathrm{H}$, Finkenzeller T, Wurth $\mathrm{S}$ et al. Individual and team performance in team-handball: A review. J Sports Sci Med 2014; 13: 808-816

[27] Brage S, Brage N, Franks PW et al. Reliability and validity of the combined heart rate and movement sensor Actiheart. Eur J Clin Nutr 2005; 59: 561-570. doi: 10.1038/sj.ejcn.1602118

[28] Ghidey W, Lesaffre E, Verbeke G. A comparison of methods for estimating the random effects distribution of a linear mixed model. Stat Methods Med Res 2010; 19: 575-600. doi: 10.1177/0962280208091686

[29] Boukhris O, Trabelsi K, Ammar A et al. A 90 min daytime nap opportunity is better than 40 min for cognitive and physical performance. Int J Environ Res Public Health 2020; 17: 4650. doi:10.3390/ijerph17134650

[30] Suppiah HT, Low CY, Choong G et al. Effects of a short daytime nap on shooting and sprint performance in high-level adolescent athletes. Int J Sports Physiol Perform 2018: 1-25. doi: 10.1123/ijspp.2018-0107

[31] Monma T, Ando A, Asanuma T et al. Sleep disorder risk factors among student athletes. Sleep Med 2018; 44: 76-81. doi: 10.1016/j. sleep.2017.11.1130

[32] Suppiah HT, Low CY, Chia M. Effects of Sport-Specific Training Intensity on Sleep Patterns and Psychomotor Performance in Adolescent Athletes. Pediatr Exerc Sci 2016; 28: 588-595. doi: 10.1123/ pes.2015-0205

[33] Dement WC. Sleep extension: getting as much extra sleep as possible. Clin Sports Med 2005; 24: 251-268. doi: 10.1016/j.csm.2004.12.014.
[34] Mah CD, Mah KE, Kezirian EJ et al. The effects of sleep extension on the athletic performance of collegiate basketball players. Sleep 2011; 34: 943-950 . doi: 10.5665/SLEEP.1132

[35] Gupta L, Morgan K, Gilchrist S. Does elite sport degrade sleep quality? A systematic review. Sports Med 2017; 47: 1317-1333. doi: 10.1007| s40279-016-0650-6

[36] Schwartz ], Simon RD. Sleep extension improves serving accuracy: A study with college varsity tennis players. Physiol Behav 2015; 151: 541-544 . doi: 10.1016/j.physbeh.2015.08.035

[37] Roberts SSH, Teo WP, Aisbett B et al. Extended sleep maintains endurance performance better than normal or restricted sleep. Med Sci Sports Exerc 2019; 51: 2516-2523. doi: 10.1249/ MSS.0000000000002071

[38] Buchheit M. Monitoring training status with HR measures: Do all roads lead to Rome? Front Physiol 2014; 5: 73. doi: 10.3389/ fphys.2014.00073

[39] Atlaoui D, Pichot V, Lacoste L et al. Heart rate variability, training variation and performance in elite swimmers. Int J Sports Med 2007; 28: 394-400. doi: 10.1055/s-2006-924490

[40] Flatt AA, Hornikel B, Esco MR. Heart rate variability and psychometric responses to overload and tapering in collegiate sprint-swimmers. J Sci Med Sport 2017; 20: 606-610. doi: 10.1016/j.jsams.2016.10.017

[41] Flatt AA, Esco MR, Nakamura FY et al. Interpreting daily heart rate variability changes in collegiate female soccer players. J Sports Med Phys Fitness 2017; 57: 907-915

[42] Baumert M, Brechtel L, Lock J et al. Heart rate variability, blood pressure variability, and baroreflex sensitivity in overtrained athletes. Clin J Sport Med 2006; 16: 412-417. doi: 10.23736/S00224707.16.06322-2

[43] Chen PC, Sattari N, Whitehurst LN et al. Age-related losses in cardiac autonomic activity during a daytime nap. Psychophysiology 2020: e13701. doi: 10.1111/psyp. 13701

[44] Natarajan A, Pantelopoulos A, Emir-Farinas $\mathrm{H}$ et al. Heart rate variability with photoplethysmography in 8 million individuals: A crosssectional study. Lancet Digit Health 2020; 2: e650-e657. doi: $10.1016 /$ S2589-7500(20)30246-6 